\title{
Genes encoding bile salt hydrolases and conjugated bile salt transporters in Lactobacillus johnsonii 100-100 and other Lactobacillus species
}

\author{
Christopher A. Elkins, Scott A. Moser and Dwayne C. Savage \\ Author for correspondence: Dwayne C. Savage. Tel: +1 865974 4015. Fax: +1 8659744007. \\ e-mail: Dsavage1@utk.edu
}

Department of Microbiology, M409

Walters Life Sciences Bldg, University of Tennessee, Knoxville, TN 37996-0845 USA

\begin{abstract}
Lactobacillus johnsonii strain 100-100 expresses two antigenically distinct conjugated bile salt hydrolases (BSH), $\alpha$ and $\beta$, that combine to form native homo- and heterotrimers. This paper reports characterization of loci within the genome that encode this capacity. A locus that encodes BSH $\beta$ (cbsH $\beta)$, a partial (cbsT1) and a complete conjugated bile salt transporter (cbsT2) was identified previously. DNA sequence analysis at this locus was extended and revealed a complete ORF for cbsT1 and no other ORFs in tandem. The three genes, cbsT1, $c b s T 2$ and $c b s H \beta$, probably constitute an operon; a putative promoter was identified upstream of cbsT1. A second locus that expresses BSH activity in strain 100-100 was identified. Sequence analysis of the clone predicted a $978 \mathrm{nt}$ ORF that did not share tandem organization with other ORFs, was similar in sequence to other BSH genes, and matched, in predicted protein sequence, the first 25 amino acids of BSH $\alpha$. A phenotypic screen for BSH activity and a genetic screen for the cbs $\mathrm{H} \beta$ locus were performed on 50 Lactobacillus isolates from humans or dairy products. Nearly all of the isolates that were positive for $c b s H \beta$ were from human sources. Variability in the BSH phenotype and cbs $\beta$ $\beta$ genotype was identified in isolates of the same species. DNA sequence was obtained and analysed from the cbsH $\beta$ locus of one human isolate, L. acidophilus strain KS-13. This organism has cbsT1, cbsT2 and cbs $\beta$ genes that are 84, 87 and $85 \%$ identical in DNA sequence to those of strain 100-100. DNA sequence identity to strain 100-100 ends in regions flanking this locus. The findings of this study suggest that BSH genes have been acquired horizontally and that BSH activity is important at some level for lactobacilli to colonize the lower gastrointestinal tract.
\end{abstract}

Keywords: intestinal microflora, probiotics, major facilitator superfamily, horizontal gene transfer, lactic acid bacteria

\section{INTRODUCTION}

The autochthonous microbiota inhabiting the lower gastrointestinal tract of animals (Savage, 1977) have evolved the ability to alter bile acids biochemically

\footnotetext{
Abbreviations: $\mathrm{BSH}$, bile salt hydrolase; $\mathrm{EF}$, extracellular factor; LDGW long-distance genome walking; LPEA, long primer extension and amplification; MFS, major facilitator superfamily.

The GenBank accession numbers for the sequences determined in this work are AF054971, AF091248 (sequences of the complete BSH operons in $L$. johnsonii strain 100-100 and L. acidophilus strain KS-13, respectively) and AF297873 (sequence of $c b s H \alpha$ ).
}

(Baron \& Hylemon, 1997). Prokaryotic organisms from several genera express proteins that catalyse hydrolysis of an amide bond between the C-24 position of the steroid moiety and an amino acid, either glycine or taurine (Savage et al., 1995; Baron \& Hylemon, 1997; Savage, 2000). This activity may affect animal health, including that of humans. For example, it is associated with growth depression in monogastric animals grown for food (Eyssen \& De Somer, 1963; Cole \& Fuller, 1974). In humans, it may contribute to colon carcinogenesis (Kay, 1981; Cheah, 1990; Kandell \& Bernstein, 1991) but may lower blood cholesterol levels (De Smet et al., 1994). Some bacterial species express enzymes 
that catalyse side-chain (sterol) transformations such as $7 \alpha$ - and $7 \beta$-dehydroxylation, $6 \beta$-dehydrogenation and desulfation (Baron \& Hylemon, 1997; Hylemon \& Harder, 1999). Bile salt hydrolysis, however, is the principal microbial reaction in that it performs a 'gatekeeping' function: bile salts must be deconjugated before sterol transformations can occur (Batta et al., 1990).

The class of microbial enzymes that catalyse deconjugation have been collectively named conjugated bile salt hydrolases (BSH, EC 3.5.1.24). These enzymes can have demonstrable specificity for tauro- versus glycoconjugates (Aries \& Hill, 1970; Gilliland \& Speck, 1977), and with the exception of one expressed by Clostridium perfringens (Kishinaka et al., 1994), are soluble cytosolic proteins (Stellwag \& Hylemon, 1976; Gopal-Srivastava \& Hylemon, 1988; Kawamoto et al., 1989; Lundeen \& Savage, 1990; Grill et al., 1995; Tanaka et al., 2000). The molecular fate of the amino acid released by deconjugation in the bacterial cell and the benefit that the organisms may derive from such activity is unclear (De Smet et al., 1995). The deconjugated product, or primary bile acid, is a toxic hydrophobic molecule that can disaggregate the cytoplasmic membrane (Thanassi et al., 1997). These molecules are not metabolized and leave the cell passively or through some unidentified bile acid export mechanism. At the physiological $\mathrm{pH}$ of the intestinal lumen, deconjugates can be actively transported through the epithelium (Wong et al., 1994; Hylemon \& Harder, 1999) and into the bloodstream of the host, or are precipitated in the faeces and excreted (Baron \& Hylemon, 1997).

BSH enzymes have been purified and characterized from Bacteroides vulgatus VI-31 (Kawamoto et al., 1989), Bacteroides fragilis subsp. fragilis (Stellwag \& Hylemon, 1976), Bifidobacterium longum BB536 (Grill et al., 1995) and SBT2928 (Tanaka et al., 2000), C. perfringens MCV 815 (Gopal-Srivastava \& Hylemon, 1988) and Lactobacillus johnsonii strain 100-100 (Lundeen \& Savage, 1990, 1992a). Genes encoding BSH activity have been cloned from Lactobacillus plantarum 80 (Christiaens et al., 1992), C. perfringens 13 (Coleman \& Hudson, 1995), L. johnsonii strain 100-100 (Elkins \& Savage, 1998) and Bif. longum SBT2928 (Tanaka et al., 2000). Genetic characterization of these loci reveals different architectures: the L. plantarum $80 \mathrm{BSH}$ transcript is monocistronic (Christiaens et al., 1992); the L. johnsonii strain 100-100 BSH gene is arranged in tandem with at least two other functionally related genes (Elkins \& Savage, 1998); and the Bif. longum SBT2928 BSH gene is coordinately regulated with at least one other gene that shares a high level of amino acid homology with glutamine synthetase adenylyltransferase $(g \ln E$; Tanaka et al., 2000). The extent of the operon in Bif. longum SBT2928 and the putative operon in L. johnsonii strain 100-100 is not known (Elkins \& Savage, 1998; Tanaka et al., 2000); DNA sequence flanking the C. perfringens 13 BSH gene has not been characterized (Coleman \& Hudson, 1995).
L. johnsonii strain 100-100 expresses high levels of BSH activity (Lundeen \& Savage, 1990). In contrast to other bacteria studied, the activity in strain 100-100 involves four enzymes that exist as homo- and heterotrimers of two antigenically distinct proteins, designated $\alpha$ and $\beta$ (Lundeen \& Savage, 1992a). A 2977 bp EcoRI genomic clone, pIN-BSH2, that expresses hydrolase activity in Escherichia coli cells has been identified and shown to encode the $\beta$ peptide (ORF3, hereafter referred to as cbsH $\beta$; Elkins \& Savage, 1998). A 651 nt partial ORF1 (hereafter referred to as $c b s T 1$ ) and a $1353 \mathrm{nt}$ complete ORF2 (hereafter referred to as $c b s T 2$ ) were encoded on the fragment $5^{\prime}$ of $c b s H \beta$. These ORFs share similarity with transport proteins of the major facilitator superfamily (MFS; Saier et al., 1999) and approximately $80 \%$ predicted amino acid sequence similarity to each other. They increase uptake of conjugated bile acids by as much as threefold over control levels when expressed in E. coli cells that were exposed to an extracellular factor (EF; Lundeen \& Savage, 1990, 1992b) produced by strain 100-100 (Elkins \& Savage, 1998).

We have characterized this locus and report here that cbsT1, cbsT2 and cbsH $\beta$ probably consitute a BSH operon. In addition, we report a second locus that encodes BSH $\alpha$. We have also assayed a collection of 50 Lactobacillus spp. for BSH activity and the $c b s H \beta$ locus. We speculate that BSH activity was acquired horizontally and implicate the activity as an important factor for colonization by lactobacilli of the gastrointestinal tract.

\section{METHODS}

Culture techniques. L. johnsonii strain 100-100 was maintained at $-80^{\circ} \mathrm{C}$ in $15 \%(\mathrm{v} / \mathrm{v})$ glycerol for cryoprotection and grown anaerobically in MRS broth or agar medium (Becton Dickinson) as previously reported (Lundeen \& Savage, 1990; Savage et al., 1995). Strains of various Lactobacillus spp. obtained from several culture collections (see Table 2) were received as lyophilates, revived anaerobically in MRS medium, and subcultured. Glycerol stocks of each strain were prepared from isolated colonies and handled identically to strain $100-100$.

Oligonucleotide primers. Primers used in this study (Table 1) were synthesized by Gibco-BRL Custom Primers Division under standard conditions.

Extension of genomic DNA sequence. Long-distance genome walking polymerase chain reaction (LDGW PCR; Min \& Powell, 1998) was used to amplify and extend DNA sequence upstream and downstream of the strain 100-100 locus in pINBSH2 (Fig. 1). Long and accurate Advantage cDNA Polymerase Mix (ADP; Clontech), nested sequence-specific primers (SSPs), oligo-dG adaptor primer (AP), and thermocycling parameters established by Min \& Powell (1998) were used. Genomic DNA was purified from strain 100-100 cells (Wizard Genomic DNA Purification Kit; Promega). LDGW was performed on genomic DNA with nested SSPs, UP-1 and UP-2, that were engineered to the upstream end of the $2977 \mathrm{bp}$ EcoRI fragment. The LDGW protocol was modified to include a second nested amplification with UP-3 that eliminated contaminating DNA species and enriched for the genomic DNA sequence of interest. DNA from the second reaction was 
Table 1. Oligonucleotide primers used in this study

\begin{tabular}{|c|c|c|c|c|}
\hline Primer & Nucleotide sequence $\left(5^{\prime}-3^{\prime}\right)^{*}$ & Strand $\dagger$ & Location & Use \\
\hline $\mathrm{AP}$ & CGAGGAATTCGGGGGGGGGGGGGG & NA & NA & LDGW, LPEA \\
\hline UP-1 & CССТАAACCAATAATTAAAAAGCCG & - & cbsT1 (768-792) & LDGW \\
\hline UP-2 & GCCGATACTAAAAATCCAGAACCATTTC & - & cbsT1 (744-771) & LDGW \\
\hline UP-3, T1-1 & CCATTTCATGTGAAGAACTTGCTTGCCGCTCC & - & cbsT1 (719-750) & LDGW, LPEA \\
\hline DN-1 & GCTACTCTTCTGGAAGCAAGACTTACTAC & + & $c b s H \beta(839-867)$ & LDGW \\
\hline DN-2 & CTACTGTAATTTTGAAGATGATTTTGAA & + & $c b s H \beta(864-891)$ & LDGW \\
\hline DN-3 & AAAGACTTATAAACTAGACGATCACAC & + & cbsH $\beta(894-920)$ & LDGW \\
\hline $\mathrm{H} \beta-1$ & AATCCTGTCCAACATCTAGATTACG & - & $c b s H \beta(52-76)$ & LPEA \\
\hline T1-2 & ACACCTTGACCAGAATAAAAGTTGAACCAACC & - & cbsT1 (360-392) & LPEA \\
\hline T1strt & CGACAATATAAGTGGgTAccGTCAAAGCTC & + & $5^{\prime}$ of $c b s T 1(64-93)$ & Cloning, pT1T2H $\beta$ \\
\hline T2strt & GGTTTTGCTATTGGTaCCCCACTATTTGGC & + & cbsT1 (1174-1203) & Cloning, $\mathrm{pT} 2 \mathrm{H} \beta$ \\
\hline $\mathrm{H} \beta$ strt & GGCTATGCCATT $\overline{\text { GGTaCcCCACTATTTGG } \neq}$ & + & cbsT2 (1171-1199) & Cloning, $\mathrm{pH} \beta$ \\
\hline $\mathrm{H} \beta$ end & CGACCAGGCTTGATgGATCCGCS & - & $3^{\prime}$ of $c b s H \beta(137-158)$ & $\begin{array}{c}\text { Cloning, } \mathrm{pT} 1 \mathrm{~T} 2 \mathrm{H} \beta \\
\mathrm{pT} 2 \mathrm{H} \beta \text { and } \mathrm{pH} \beta\end{array}$ \\
\hline $\mathrm{H} \beta 675 \mathrm{a}$ & ATGTGTACTGGTTTAAGATTC & + & $c b s H \beta(1-21)$ & PCR screen for $c b s H \beta$ \\
\hline $\mathrm{H} \beta 675 \mathrm{~b}$ & GGCTGGAATGCTGTCACCAGGCAGACC & - & $c b s H \beta(649-675)$ & PCR screen for $c b s H \beta$ \\
\hline $\mathrm{La}-\mathrm{H} \beta \mathrm{a}$ & GCTATTGGTaCcCCATTATTCGG $\neq \|$ & + & cbsT2 (1177-1199) & Cloning, $\mathrm{pLaH} \beta$ \\
\hline $\mathrm{La}-\mathrm{H} \beta \mathrm{b}$ & GTCATTTGGGAgGTAcCTGTTGAATCAGG规 & - & $3^{\prime}$ of $c b s H \beta(163-191)$ & Cloning, $\mathrm{pLaH} \beta$ \\
\hline
\end{tabular}

*Lower-case nucleotides indicate base substitutions in the sequence used to create restriction enzyme sites (underlined).

† Primers were engineered to the coding strand $(+)$ or the noncoding strand $(-)$ of the $c b s T 1$, cbsT2 and cbsH $\beta$ locus in L. johnsonii strain 100-100. NA, Not applicable; AP primer duplicated from procedure outlined by Min \& Powell (1998).

$\ddagger K p n$ I site underlined.

$\$ B a m H I$ site underlined.

\| Primers were engineered to the cbsH $\beta$ locus in L. acidophilus strain KS-13.

purified (Wizard PCR Preps DNA Purification System) and sequenced with a fourth nested SSP and walking primers. The sequence was reconfirmed by directly amplifying genomic DNA with UP-1 and a primer engineered to the downstream end of the new sequence. The same strategy was applied to DNA sequence downstream of the cloned locus with primers, DN-1, DN-2 and DN-3. DNA sequence upstream and downstream of a 675 bp PCR amplified locus in L. acidophilus strain KS-13 was also obtained with LDGW (primers not shown).

RNA transcript analysis. Strain $100-100$ is known to express BSH activity upon entry into stationary phase (Lundeen \& Savage, 1990). Whole-cell RNA was harvested from stationary-phase cells (TRI Reagent; Molecular Research Center). Approximately $2 \mathrm{ml}$ of cells were incubated with a $120 \mu \mathrm{l}$ solution of $10 \mathrm{mg} \mathrm{ml}^{-1}$ lysozyme (Sigma) $/ 10 \mathrm{mg} \mathrm{ml}^{-1}$ lysostaphin (Sigma) for $1 \mathrm{~h}$ at $37^{\circ} \mathrm{C}$ prior to RNA isolation. Analysis of the mRNA that encodes $c b s H \beta$ was performed with long primer extension and amplification (LPEA) PCR developed in our laboratory as a hybrid protocol of rapid amplification of cDNA Ends (RACE; Edwards et al., 1995) and LDGW (Min \& Powell, 1998). The method involves use of Moloney Murine Leukaemia Virus-reverse transcriptase (M-MLV RT) and ADP to extend over long distances $(>7 \mathrm{~kb})$ on template nucleic acids. A microcentrifuge tube containing approximately $2 \mu \mathrm{g}$ strain 100-100 RNA and $50 \mathrm{pmol}$ primer $\mathrm{H} \beta-1$ was adjusted to a final volume of $15 \mu \mathrm{l}$ with distilled $\mathrm{H}_{2} \mathrm{O}$, heated to $70{ }^{\circ} \mathrm{C}$ for $5 \mathrm{~min}$, and immediately cooled on ice. Two hundred units of M-MLV RT (Promega), $5 \mu$ of $5 \times$ M-MLV RT reaction buffer, 10 pmol of each dNTP (UltraPure; Clontech) and 25 units of rRNasin Ribonuclease inhibitor (Promega) were added to a final volume of $25 \mu \mathrm{l}$. The tube was incubated at a stringent temperature for the M-MLV $\mathrm{RT}$ of $42^{\circ} \mathrm{C}$ for $60 \mathrm{~min}$. DNA was purified (Wizard PCR Preps DNA Purification System) from two such RT reactions and concentrated to approximately $15 \mu \mathrm{l}$. A dCTP nucleotide tail was added to the 3' end of the cDNA. Two successive, nested PCR reactions were accomplished as described by Min \& Powell (1998) with tailed cDNA, AP and T1-1 (see Fig. 1) and then template DNA produced in the first reaction, T1-2 and AP. Total DNA from the second PCR reaction was purified and sequenced with T1-2. The DNA complement of the nucleotide adjacent to the oligo-dC tail was identified as the putative transcriptional start site.

Recombinant DNA methods. Plasmid vector pSportI (GibcoBRL) was used in all recombinant DNA procedures. Three constructs that harbour strain 100-100 genomic loci encoding cbsT1-cbsT2-cbsH $\beta$, cbsT2-cbsH $\beta$ or $c b s H \beta$ were cloned under control of the pSportI lac-inducible promoter. Primers containing engineered $K p n I$ sites, T1strt, T2strt and $\mathrm{H} \beta$ strt, were constructed $5^{\prime}$ of $c b s T 1, c b s T 2$ and $c b s H \beta$ respectively and used with a primer containing an engineered $\mathrm{BamHI}$ site $3^{\prime}$ of $c b s H \beta, \mathrm{H} \beta$ end. Genomic DNA was amplified by PCR, digested with KpnI and BamHI (Promega), and cloned into identically digested vector DNA with T4 DNA ligase (Promega). The chimeras were designated pT1T2H $\beta, \mathrm{pT} 2 \mathrm{H} \beta$ and $\mathrm{pH} \beta$, respectively, and transformed into E. coli $\mathrm{DH} 5 \alpha$ cells. Constructs were identified by blue/white colony screening on Luria-Bertani (LB) medium containing $100 \mu \mathrm{g}$ ampicillin (Amp) ml ${ }^{-1}, 0.5 \mathrm{mM}$ IPTG and $40 \mu \mathrm{g} \mathrm{X-Gal} \mathrm{ml^{-1 }}$ (LB-Amp-IPTG-X-Gal). Plasmid DNA for each construct was purified (Wizard Plus Minipreps DNA Purification Kit) and 


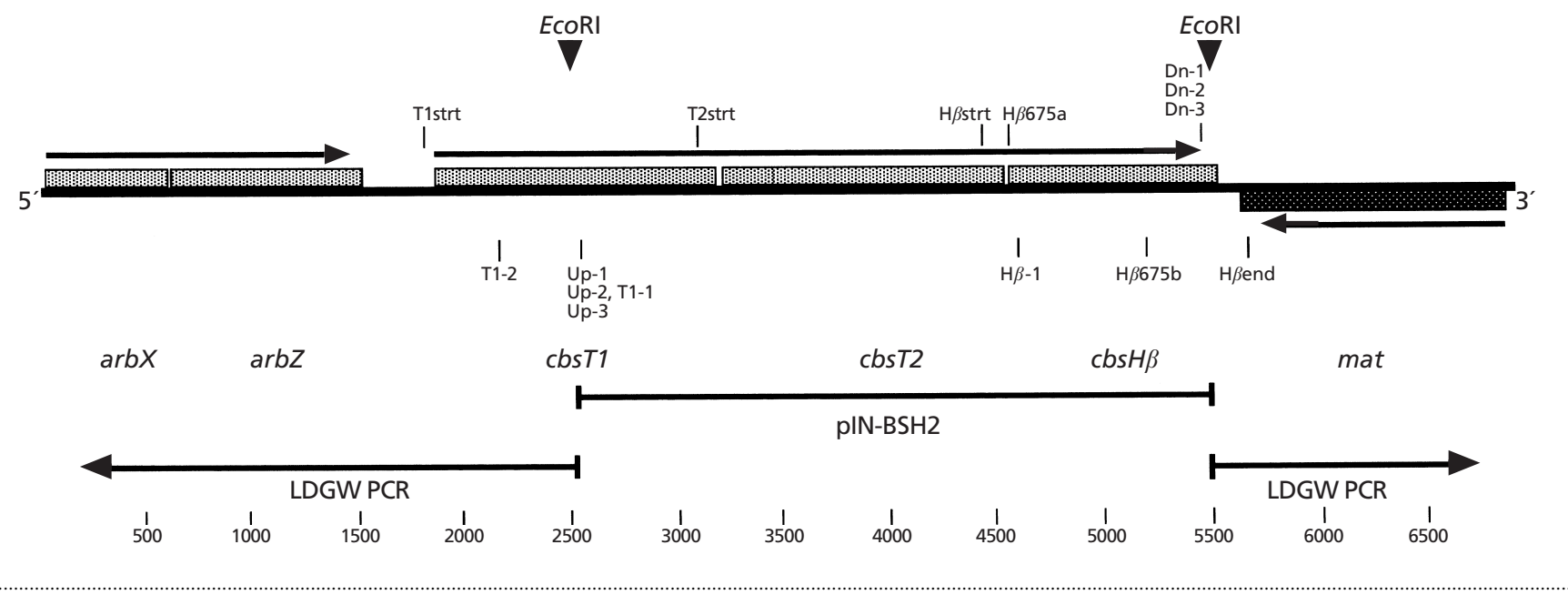

Fig. 1. LDGW PCR (Min \& Powell, 1998) was used to amplify strain 100-100 genomic DNA $5^{\prime}$ and $3^{\prime}$ of an EcoRI cloned locus, pIN-BSH2 (Elkins \& Savage, 1998), and revealed a complete ORF for cbsT1 and three additional ORFs: arbX, arbZ and mat. BLAST analysis identified high similarity of the three ORFs to glycosyltransferases, phospho- $\beta$-glycosidases and group II intron encoding maturases, respectively. Locations of primers used in this study (Table 1) are shown. A scale in bp is shown at the bottom.

sequenced to verify content and orientation. To clone and express the predicted BSH gene from L. acidophilus strain KS13, chromosomal DNA was purified from strain KS-13 cells and PCR-amplified with primers $\mathrm{La}-\mathrm{H} \beta \mathrm{a}$ and $\mathrm{La}-\mathrm{H} \beta \mathrm{b}$. The fragment was cloned at the KpnI site of pSportI. Transformants were screened for BSH activity.

Generation of an L. johnsonii strain 100-100 genomic library. A genomic library of strain $100-100$ was created with pSportI and screened for BSH activity. Chromosomal DNA was purified from strain 100-100 cells and partially digested with Sau3AI (Promega). The fragments were cloned at the BamHI site of pSportI in E. coli DH5 $\alpha$ cells. The library was screened for clones expressing BSH activity.

DNA sequencing and analysis. DNA generated from LDGW and LPEA PCR, direct amplified DNA, and cloned DNA was sequenced by the ABI Prism automated fluorescent method (Molecular Biology Resource Facility, University of Tennessee, Knoxville). Direct amplified DNA and cloned DNA were sequenced bidirectionally using universal forward and reverse primers and/or synthesized oligonucleotide walking primers. DNA sequence was analysed for start and stop codons and translated into predicted amino acid sequence with MacVector. ORFs were putatively identified from similarity with published sequences using the advanced basic local alignment search tool (BLAST; NIH website).

Assay for BSH activity. Transformed E. coli $\mathrm{DH} 5 \alpha$ cells were screened for the ability to hydrolyse conjugated bile acids with an agar plate assay (Dashkevicz \& Feighner, 1989). Cultures were streaked or replica plated onto MRS agar containing $0.5 \%$ taurodeoxycholic acid (Sigma), a conjugated bile acid, $100 \mu \mathrm{g} \mathrm{Amp} \mathrm{ml} \mathrm{m}^{-1}$ and $0.5 \mathrm{mM}$ IPTG (MRS-TDCA-AmpIPTG). Cultures were incubated anaerobically for 24-48 h. $\mathrm{BSH}$ activity was detected when a halo of deoxycholic acid precipitated around colonies in the medium.

Screen for $\boldsymbol{c b s} \boldsymbol{H} \boldsymbol{\beta}$. Coleman \& Hudson (1995) identified conserved amino acid motifs in cloned BSHs by aligning predicted protein sequences. A motif at the amino terminus and a motif centred around residue 225 were selected and used to construct primers $\mathrm{H} \beta 675 \mathrm{a}$ and $\mathrm{H} \beta 675 \mathrm{~b}$, respectively. The primers were engineered to $c b s H \beta$ sequence in strain $100-100$ and encompass a region of approximately $675 \mathrm{bp}$. The primers were used in standard PCR reactions to amplify genomic DNA from other lactobacilli.

\section{RESULTS}

\section{Genomic analysis of the cbsH $\beta$ locus}

Genomic DNA sequence upstream and downstream of the cloned locus in pIN-BSH2 was extended and analysed. DNA fragments of approximately 2000$2500 \mathrm{bp}$ were amplified in both directions by LDGW (Fig. 1). Sequence upstream of the locus yielded a complete ORF of $1356 \mathrm{nt}$ for $c b s T 1$ that is $75 \%$ identical and $86 \%$ similar in predicted amino acid sequence to cbsT2. A putative Shine-Dalgarno 16S rRNA sequence was identified that is centred 8-13 nt upstream of the ATG start site for $c b s T 1$. Advanced BLAST analysis of CbsT1 and CbsT2 revealed similarities to several families of the MFS (Saier et al., 1999). The highest scoring, with approximately $42 \%$ similarity to the first 209 and 223 amino acids of CbsT1 and CbsT2 (6 and $10 \%$ gaps), respectively, were oxalate:formate antiporters from Archaeoglobus fulgidus and Oxalobacter formigenes (Abe et al., 1996). MacVector also predicted two additional clustered ORFs 305 nt upstream of $c b s T 1$ on the coding strand. These ORFs are highly similar in spatial arrangement and predicted amino acid sequence to two coordinately regulated genes, $a r b X$ and $a r b Z$, in L. delbrueckii subsp. lactis that encode a putative glycosyltransferase and a phospho- $\beta$-glycosidase, respectively (Weber et al., 1998). An ORF of $1245 \mathrm{nt}$ was identified 119 nt downstream of $c b s H \beta$, in the opposite orientation. BLAST analysis putatively identified the ORF, designated mat, as a group II intron encoding maturase (Fig. 1; Edgell et al., 2000). 
(a)

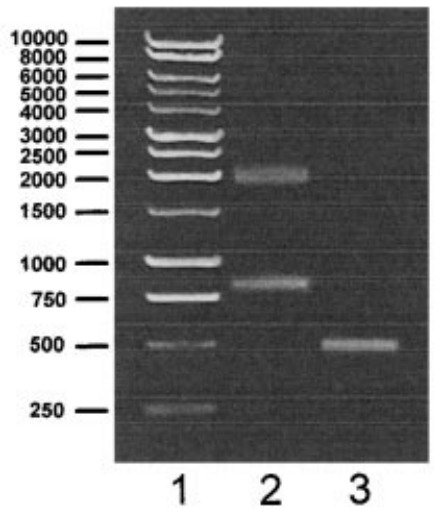

(b)

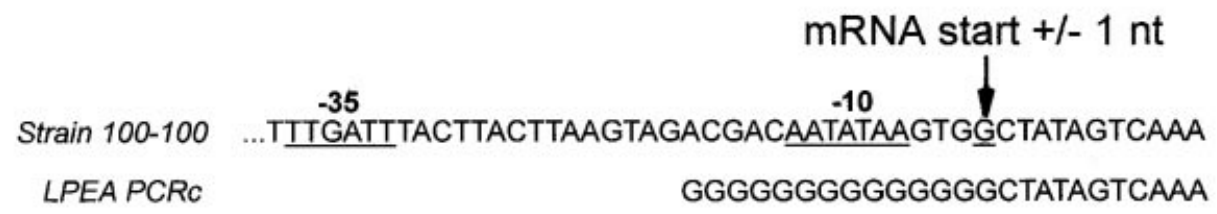

Strain 100-100 GCTCACGTGTTTAATAATATATAACTTATAAAGCTAATAGTATTTTATCAGA

LPEA PCRC GCTCACGTGTTTAATAATATATAACTTATAAAGCTAATAGTATTTTATCAGA

Strain 100-100 AAGAAGAGAGATACGATGTCAAACGATCTACCAAGCGATGGGCACAAG...

LPEA PCRC AAGAAGAGAGATACGATGTCAAACGATCTACCAAGCGATGGGCACAAG...

cbST1 ORF

Fig. 2. (a) Ethidium bromide-agarose gel electrophoresis of LPEA PCR products. Lane 1, $1 \mathrm{~kb}$ DNA ladder (Promega) with fragment sizes in $\mathrm{bp}$; lane 2, DNA products from first, nested PCR reaction containing oligo-dC tailed CDNA, T1-1 and AP; lane 3, products from the second, nested PCR reaction containing DNA template from lane 2, T1-2 and AP. (b) The complement of the DNA sequence from second, nested LPEA PCR reaction (LPEA PCRC; panel a, lane 3 ) is identical to the genomic sequence from strain 100-100. A transcriptional start site (arrow) and putative promoter (underlined) were identified.

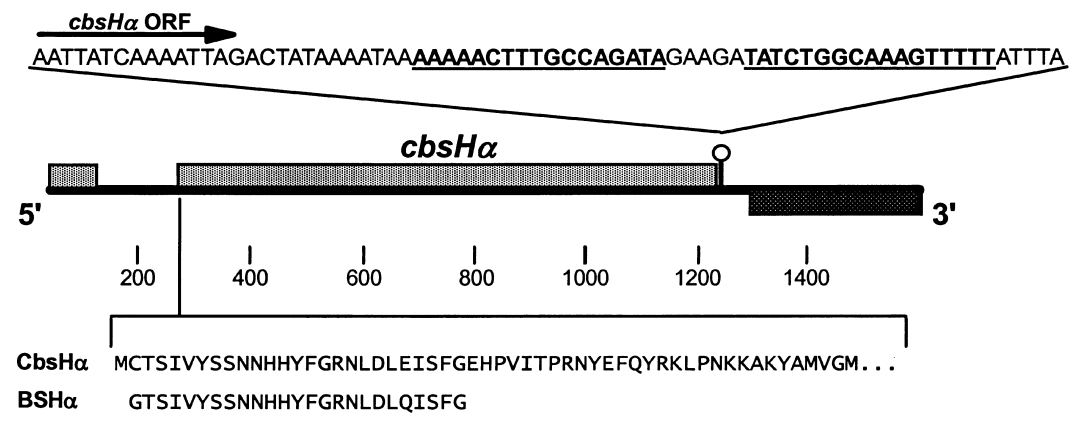

Fig. 3. A BSH-positive genomic clone from strain 100-100, pBSH2, with predicted ORFs. The predicted amino acid sequence of $c b s \mathrm{H} \alpha$ matched the protein sequence of purified $\mathrm{BSH} \alpha$ (Lundeen \& Savage, 1992a). An inverted repeat (underlined) was identified downstream of the $\mathrm{cbs} H \alpha$ stop codon.

\section{BSH operon in strain 100-100}

LPEA produced long cDNA that extended to the $5^{\prime}$ terminus of the mRNA species complementary to primer $\mathrm{H} \beta-1$. This cDNA was amplified with AP and nested primers, T1-1 and T1-2, in two successive nested LDGW PCR reactions. The first PCR reaction produced two visible bands of approximately 2 and $0.8 \mathrm{kbp}$ when analysed by $1 \%$ agarose gel electrophoresis (Fig. 2a, lane 2). The second, nested reaction produced a single fragment of approximately $0.5 \mathrm{kbp}$ (Fig. 2a, lane 3 ). Considering the respective placement of T1-1 and T1-2, a decrease of approximately $0.3 \mathrm{kbp}$ from 0.8 to $0.5 \mathrm{kbp}$ is proportional to and consistent with genomic DNA sequence from strain 100-100. We believe that the fragment of $2 \mathrm{kbp}$ (Fig. 2a, lane 2) is an artifact. LDGW PCR is known to produce artifacts of unrelated sequence that can be eliminated, in our experience, in subsequent nested amplifications with SSPs. Alternatively, or in addition, the $2 \mathrm{kbp}$ fragment could have been amplified from a similar primer-binding site for T1-1 that is found within $c b s T 2$. Purified DNA from the second reaction was sequenced with T1-2 and matched identically the
DNA sequence from the genome of strain 100-100 directly downstream of the T1-2 binding site (Fig. 2b). The complement of the DNA sequence extended $78 \mathrm{nt}$ upstream of $c b s T 1$ and was tailed with a GS stretch. Sequences centred approximately -10 and -35 of the transcriptional start site, AATATAA and TTGATT respectively, were identified that resemble $E$. coli -10 and -35 consensus promoter sequences, TATAAT and TTGACA respectively, that are recognized by $\sigma^{70}$ (Fig. $2 \mathrm{~b})$. Expression analysis of $c b s H \beta$ was undertaken with three clones, $\mathrm{pT} 1 \mathrm{~T} 2 \mathrm{H} \beta, \mathrm{pT} 2 \mathrm{H} \beta$ and $\mathrm{pH} \beta$. All three clones expressed BSH activity when transformed into E. coli DH5 $\alpha$ cells incubated anaerobically on MRSTDCA-Amp-IPTG plates (data not shown).

\section{Identification of $c b s H \alpha$}

A genomic library of strain 100-100 was prepared in $E$. coli and colonies were screened anaerobically on MRSTDCA-Amp-IPTG plates. Two colonies, designated pBSH1 and pBSH2, produced a halo of deoxycholic acid. DNA sequence analysis of $\mathrm{pBSH} 1$ revealed a fragment encoding the cbsH $\beta$ locus. The second BSH- 
Table 2. BSH phenotype and cbsH $\beta$ genotype of strains of Lactobacillus spp.

\begin{tabular}{|c|c|c|c|c|}
\hline Species & Strain* & Source & $\begin{array}{c}\mathrm{BSH} \\
\text { activity } \dagger\end{array}$ & $\begin{array}{l}c b s H \beta \\
\text { locus } \neq\end{array}$ \\
\hline \multirow[t]{9}{*}{ L. acidophilus } & ATCC 4356 & Human & + & - \\
\hline & ATCC 4357 & Human & + & - \\
\hline & ATCC 53544 & Human infant, rectal swab & + & - \\
\hline & BCCM 8151 & Acidophilus milk & 0 & - \\
\hline & JCM 1028 & Human intestine & + & + \\
\hline & JCM 1034 & Human intestine & + & + \\
\hline & JCM 1229 & Human intestine & $(+)$ & - \\
\hline & $\begin{array}{l}\text { NCSK } 1070 \\
\text { (ATCC 700396) }\end{array}$ & Unknown & + & - \\
\hline & PI KS-13 & Human & + & + \\
\hline \multirow[t]{4}{*}{ L. brevis } & ATCC 14869 & Human faeces & - & $(-)$ \\
\hline & BCСM 7944 & Human faeces & + & - \\
\hline & BCCM 11998 & Starter from dairy & + & - \\
\hline & BCCM 18022 & Zabady (yogurt) & + & - \\
\hline \multirow[t]{2}{*}{ L. buchneri } & JCM 1068 & Human intestine & - & - \\
\hline & JCM 1069 & Human intestine & + & - \\
\hline \multirow{3}{*}{$\begin{array}{l}\text { L. delbrueckii subsp. } \\
\text { bulgaricus }\end{array}$} & ATCC 11842 & Bulgarian yogurt & 0 & + \\
\hline & BCCM 12168 & Homemade yogurt & 0 & - \\
\hline & DSMZ 20080 & Yogurt & 0 & $(-)$ \\
\hline \multirow[t]{4}{*}{ L. fermentum } & ATCC 11976 & Intestine of infant & $(+)$ & - \\
\hline & ATCC 23271 & Human intestine & - & - \\
\hline & ВССМ 6902 & Fermented beets & - & - \\
\hline & DSMZ 20050 & Unknown & - & - \\
\hline \multirow[t]{4}{*}{ L. gasseri } & ATCC 19992 & Faeces & - & + \\
\hline & BCСM 9203 & Human & + & + \\
\hline & JCM 1025 & Human intestine & + & + \\
\hline & NCSK 99 & Unknown & $(+)$ & + \\
\hline \multirow[t]{2}{*}{ L. johnsonii } & JCM 1022 & Human intestine & + & + \\
\hline & JCM 8791 & Human faeces & + & + \\
\hline \multirow[t]{3}{*}{ L. plantarum } & BCCM 18021 & Milk & $(+)$ & - \\
\hline & BCCM 18027 & Laban rayeb & $(+)$ & - \\
\hline & BCCM 18035 & Milk & - & - \\
\hline
\end{tabular}

*ATCC, American Type Culture Collection; JCM, Japanese Collection of Microorganisms; BCCM, Belgian Co-oridinated Collections of Microorganisms; DSMZ, Deutsche Sammlung von Mikroorganismen und Zellkulturen GmbH (German Collection of Microorganisms and Cell Cultures); NCSK, North Carolina State University (Todd Klaenhammer); PI, Principal Investigator (our laboratory).

†From Moser \& Savage (2001). Lactobacillus strains were streaked on MRS plates containing 0.5\% taurodeoxycholic acid (MRS-TDCA). +, a halo of deoxycholic acid precipitate around bacterial growth (Dashkevicz \& Feighner, 1989); (+), weak halo; -, no halo present; 0, no growth on MRSTDCA plates.

$\ddagger$ The $c b s H \beta$ locus was screened with oligonucleotide primers $\mathrm{H} \beta 675 \mathrm{a}$ and $\mathrm{H} \beta 675 \mathrm{~b}$ using standard PCR. + , a single 675 bp PCR product; -, no amplification of genomic DNA; $(-)$, results negative $-\mathrm{a}$ ladder of bands was obtained.

positive clone, pBSH2, contained a $1610 \mathrm{bp}$ DNA fragment from the genome of strain 100-100 encoding a complete ORF of $978 \mathrm{nt}$ and two putative partial ORFs of 123 and $315 \mathrm{nt}$. Two partial ORFs were located $229 \mathrm{nt}$ upstream and $56 \mathrm{nt}$ downstream of the complete ORF, respectively (Fig. 3). Advanced BLAST analysis of the complete ORF displayed sequence similarities (DNA and predicted amino acid) to all cloned $\mathrm{BSH}$ genes (Christiaens et al., 1992; Coleman \& Hudson, 1995;
Elkins \& Savage, 1998; Tanaka et al., 2000); the BSH gene from L. plantarum 80 (Christiaens et al., 1992) scored the highest. The partial ORFs did not display strong sequence similarity to any known genes. Microsequencing of the first 25 amino acids of the $\alpha$ hydrolase $(\mathrm{BSH} \alpha)$ was done previously on purified protein blotted onto PVDF membranes (Lundeen \& Savage, 1992a). The predicted amino acid sequence from the amino terminus of the complete ORF, designated $c b s \mathrm{H} \alpha$, was 


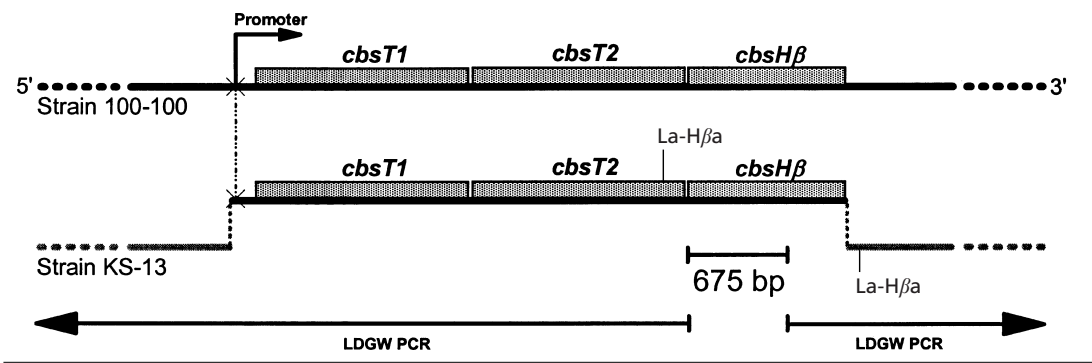

Fig. 4. The sequence of the $c b s H \beta$ locus in
the genome of $L$. acidophilus strain KS-13
was generated by standard (675 bp) and
LDGW PCR (Min \& Powell, 1998). The DNA
sequence identity to strain 100-100 ( $85 \%$ )
ends 26 nt upstream of the putative
promoter and immediately downstream of
the $c b s H \beta$ stop codon.

virtually identical (23/25 amino acids) to the $\mathrm{BSH} \alpha$ sequence (Fig. 3). DNA sequence centred approximately 8 to $13 \mathrm{nt} 5^{\prime}$ of $c b s H \alpha$, TAAGGAGGAAT, closely matched the Shine-Dalgarno 16S rRNA sequence of TAAGGAGGTGA. An inverted repeat capable of forming a stem-loop structure was identified 12 nt $3^{\prime}$ of the stop codon of this gene (Fig. 3).

\section{Survey of 50 Lactobacillus isolates for the cbsH $\beta$ locus}

Fifty strains of Lactobacillus species from humans or dairy products were obtained from culture banks and screened for BSH activity and the $c b s H \beta$ locus (Table 2). Genomic DNA from each was screened using standard PCR with primers $\mathrm{H} \beta 675 \mathrm{a}$ and $\mathrm{H} \beta 675 \mathrm{~b}$. Ten isolates screened positive for $c b s H \beta$. Nine of these isolates were cultured from human sources and belong to the species acidophilus, gasseri and johnsonii. Only one dairy isolate from Bulgarian yogurt, L. delbrueckii subsp. bulgaricus, screened positive for the locus. Some isolates from the same species displayed a variable BSH phenotype as reported by Moser \& Savage (2001), and $c b s H \beta$ genotype. Strains of species [number screened] amylovorus [2], crispatus [3], curvatus subsp. curvatus [1], kefiri [1], maltaromicus [1], paracasei subsp. paracasei [3], reuteri [2], rhamnosus [3], ruminis [1], undetermined [2] were screened for $c b s H \beta$, were negative and were therefore not included in Table 2.

\section{cbs $\mathrm{H} \beta$ locus in L. acidophilus strain KS-13}

Strain KS-13, a human isolate from our laboratory, screened positive for the $c b s H \beta$ locus (Table 2) and was chosen for an extended genetic study. Primers H $\beta 675$ a and $\mathrm{H} \beta 675 \mathrm{~b}$ amplified a single fragment of approximately $675 \mathrm{bp}$ from the genome of strain KS-13 that was $85 \%$ similar in DNA sequence to that of $c b s H \beta$ of strain 100-100. DNA fragments flanking this region in strain KS-13 were obtained by LDGW and sequenced. The composite DNA sequence was analysed with MacVector and revealed an identical genomic architecture to the operon in strain 100-100 (Fig. 4). Three tandem ORFs of 1344, 1353 and $948 \mathrm{nt}$ were predicted that displayed 84, 87 and $85 \%$ DNA sequence identity and 95, 95 and $97 \%$ predicted amino acid sequence similarity to cbsT1, cbsT2 and $c b s H \beta$ of strain 100-100, respectively. Intergenic regions of $28 \mathrm{nt}(c b s T 1-c b s T 2)$ and $21 \mathrm{nt}$ (cbsT2$c b s H \beta)$ in strain KS-13 compare with $27 \mathrm{nt}$ and $19 \mathrm{nt}$ in strain 100-100 (Elkins \& Savage, 1998), respectively
(Fig. 4). Primers $\mathrm{La}-\mathrm{H} \beta \mathrm{a}$ and $\mathrm{La}-\mathrm{H} \beta \mathrm{b}$, which contain engineered $K p n \mathrm{I}$ sites, were used to amplify and clone the predicted $c b s H \beta$ gene in strain KS-13. E. coli DH5 $\alpha$ cells were transformed with a pSportI plasmid construct containing this ORF. Colonies from such cells precipitated deoxycholic acid in the surrounding medium when grown anaerobically on MRS-TDCA-Amp-IPTG plates.

\section{DISCUSSION}

We have demonstrated that BSH activity is encoded by two separate loci on the genome of L. johnsonii strain 100-100. One locus encodes $c b s H \beta$ and is part of an operon of three genes related in function. To our knowledge, this is the first report of a complete $\mathrm{BSH}$ operon. Unlike the partial operon in Bif. longum SBT2928 (Tanaka et al., 2000), cbsH $\beta$ is encoded with functionally related genes. The second locus encodes another $\mathrm{BSH}, c b s H \alpha$, which is not arranged in tandem with other ORFs. This discovery is novel since other enteric bacteria are known to encode only one BSH (Stellwag \& Hylemon, 1976; Gopal-Srivastava \& Hylemon, 1988; Kawamoto et al., 1989; Christiaens et al., 1992; Kishinaka et al., 1994; Coleman \& Hudson, 1995; Grill et al., 1995; Tanaka et al., 2000).

The BSH operon of strain 100-100 contains two genes, $c b s T 1$ and $c b s T 2$, that share a high level of similarity in their DNA and predicted amino acid sequence. This finding suggests that the genes are duplicates. Functional studies have shown that $c b s T 2$ and partial $c b s T 1$, when expressed in E. coli, increase uptake of conjugated bile acids (Elkins \& Savage, 1998). These genes, while demonstrating putative topologies characteristic of MFS transporters (Saier et al., 1999), do not display impressive sequence similarity, as identified by BLAST, to any known genes. Members of several subfamilies of the MFS provide the closest sequence similarity. In support of our observations, M. Saier and colleagues have phylogenetically classified $c b s T 1$ and $c b s T 2$ as members of a new subfamily of the MFS called the conjugated bile salt transporter family (BST) (Saier et al., 1999; http://www-biology.ucsd.edu/ msaier/transport/ titlepage.html).

We were able to produce and amplify cDNA from strain 100-100 using primers engineered to $c b s H \beta$ and $c b s T 1$. LPEA produced one fragment of $0.5 \mathrm{kbp}$ that was identical in sequence to genomic DNA from the upstream end of cbsT1. This sequence extended $78 \mathrm{nt}$ 
upstream of the start site of $c b s T 1$. We conclude that $c b s T 1, c b s T 2$ and $c b s H \beta$ are probably encoded polycistronically. The expression analysis with $c b s H \beta$ supported that conclusion. When expressed under lacinducible promotion, clones containing cbsT1-cbsT2$c b s H \beta$ or $c b s T 2-c b s H \beta$ produced precipitates of deoxycholic acid in the medium. Therefore, cbsT1 and cbsT2 probably do not contain mRNA termination sequences, at least not ones that are recognized by E. coli. However, further expression evidence and DNA motif analysis are necessary to convincingly demonstrate the polycistronic expression of the three genes.

The genetic analysis of BSH activity supports protein characterization studies. Strain 100-100 produces two hydrolases, $\alpha$ and $\beta$, that combine to form homo- and heterotrimeric complexes. Purified $\alpha$ and $\beta$ hydrolases have estimated molecular masses of 42 and $38 \mathrm{kDa}$, respectively (Lundeen \& Savage, 1992a). The calculated molecular masses of the BSH from the Sau3AI genomic clone (complete ORF) and $c b s H \beta$ are 36667 and 34916 $\mathrm{Da}$, respectively. In addition to having the larger molecular mass, the BSH from the genomic clone had an amino-terminal sequence that matches, with only two differences, the first 25 amino acids of the $\alpha$ hydrolase (Lundeen \& Savage, 1992a). The observed differences in amino acid sequence are probably due to problems in the peptide sequencing. To prepare the $\alpha$ peptide for sequencing, it was hydrolysed with $6 \mathrm{M} \mathrm{HCl}$ and derivatized with phenylisothiocyanate as previously reported (Lundeen \& Savage, 1992a). Under such conditions, Cys cannot be resolved, and Glu and Gln cannot be distinguished during sequencing. Such problems undoubtedly explain the discrepancies between the chemical peptide sequence and the peptide sequence inferred from the nucleotide sequence. We conclude, therefore, that the complete 978 nt ORF from the Sau3AI genomic clone encodes $\mathrm{BSH} \alpha$ and designate this ORF $c b s H \alpha$. We also conclude that the known genetic elements responsible for BSH activity in strain 100-100 have been identified and cloned.

$\mathrm{CbsH} \alpha$ shares the highest amino acid sequence similarity with the BSH from L. plantarum 80 (Christiaens et al., 1992). Similar to the BSH in strain $80, c b s H \alpha$ is not arranged in tandem with other ORFs and contains an inverted repeat $3^{\prime}$ of the stop codon. The BSH protein(s), however, has not been isolated or characterized from strain 80 (Christiaens et al., 1992). We suggest that the strain may also contain a second hydrolase locus encoding additional functionally related genes if the activity was acquired horizontally. We also tentatively conclude that $c b s \mathrm{H} \alpha$, similar to the $\mathrm{BSH}$ gene from 80 , is encoded monocistronically.

Our phenotypic and genetic screens suggest that BSH activity was acquired horizontally in lactobacilli. Lactobacilli within the species acidophilus, brevis, buchneri, fermentum, gasseri and plantarum express a variable BSH phenotype. PCR screening within L. acidophilus and L. delbrueckii subsp. bulgaricus also produced variable results. Nearly all $(9 / 10)$ of the $c b s H \beta$-positive isolates were from human sources, which suggests that this locus is important for persistent colonization of the gastrointestinal tract. Taken in aggregate, these results suggest that the $\mathrm{BSH}$ phenotype and genotype is variable within a given species. It is not known whether BSH genes and related genes are encoded on mobile genetic elements. It is known, however, that the BSH gene from Bif. longum SBT2928 is flanked by inverted repeats (Tanaka et al., 2000). It is interesting to note, furthermore, that strain 100-100 encodes a maturase, mat, downstream of $c b s H \beta$. Although primary literature on bacterial maturases is scarce, the enzymes have endonuclease and reverse transcriptase activity that faciliates movement and splicing of cDNA into the genome in a process known as retrohoming (Edgell et al., 2000). Group II maturases are encoded by self-contained ORFs with independent promoters. Most group II maturases are inserted in or associated with mobile genetic elements (Edgell et al., 2000). The putative maturase in strain 100-100 is located complementary to but in close proximity with DNA encoding the BSH operon.

A BSH operon identical in genomic architecture to $L$. johnsonii strain 100-100 is conserved in L. acidophilus strain KS-13. DNA sequence identity to strain 100-100 ends immediately upstream of the putative promoter and downstream of $c b s H \beta$. We suggest that this locus forms a genomic mobile element. L. acidophilus is one of only two organisms that respond to an EF of unknown composition produced by strain 100-100 (Lundeen \& Savage, 1992b). Unlike other BSH systems, hydrolase activity in strain 100-100 is increased by as much as three- to fivefold within 20 min after conjugated bile acids are added to suspensions of stationary-phase cells. The increase is due to induction of a soluble extracellular molecule and not enzymic or regulatory proteins (Lundeen \& Savage, 1992b). EF also enhances uptake of conjugated bile acids in E. coli cells expressing cbsT2 and partial $c b s T 1$ from strain 100-100 (Elkins \& Savage, 1998). Therefore, the transporters in strain KS-13 are related to the bacterium's ability to respond to EF.

The conserved DNA sequence upstream of $c b s T 1$ in strain KS-13 supported our data on the putative promoter for the $c b s H \beta$ operon in strain 100-100. A region of approximately $135 \mathrm{nt}$ upstream from $c b s T 1$ should be conserved in strain KS-13 if it is important for promotion and transcription of the genes. Lundeen \& Savage (1990) demonstrated at a physiological level that induction of BSH expression corresponds with entry of strain 100100 into stationary phase. LPEA analysis supported this conclusion as well. There are no conserved operator/ enhancer elements since sequence identity between strain KS-13 and 100-100 ends upstream of the -35 promoter element. Thus, the RNA analysis supports simple sigma-dependent stationary-phase induction of BSH activity.

We have cloned the genetic determinants for BSH activity in L. johnsonii strain 100-100 and have extended the study into other Lactobacillius species. In strain 100100, two genetic loci encode BSH activity. The $c b s H \beta$ 
locus is an operon of three genes encoding two bile-acidrelated functions: hydrolysis and transport. This locus is conserved in other Lactobacillus species, specifically human isolates. Within some species, however, $\mathrm{BSH}$ activity and the $c b s H \beta$ locus were not constant traits. $L$. acidophilus strain KS-13 encoded the $c b s H \beta$ locus but DNA sequences flanking the three-gene operon were not conserved. Our data support a hypothesis that BSH activity is important at some level for colonization of the gastrointestinal tract and that BSH genes were acquired horizontally. Bile acid transport by contrast could be studied. Is there a bile acid exporter or is export activity catalysed concomitantly with import? What is the function of EF in bile acid transport? Experiments designed to identify the energy source and kinetics of transport may provide functional information that supports the phylogenetic assignment of $c b s T 1$ and cbsT2 into a new family of the MFS. This information, once obtained, may lead to a working model for BSH activity in enteric bacteria.

\section{ACKNOWLEDGEMENTS}

We thank Neil Quigley for performing the automated DNA sequencing and George Knauer, III, for designing primers $\mathrm{LjH} \beta 675 \mathrm{a}$ and $\mathrm{LjH} \beta 675 \mathrm{~b}$.

This research was funded by the University of Tennessee and the National Dairy Council.

\section{REFERENCES}

Abe, K., Ruan, Z.-S. \& Maloney, P. C. (1996). Cloning, sequencing, and expression in Escherichia coli of OxlT, the oxalate: formate exchange protein of Oxalobacter formigenes. J Biol Chem 271, 6789-6793.

Aries, V. \& Hill, M. J. (1970). Degradation of steroids by intestinal bacteria. Biochim Biophys Acta 202, 526-534.

Baron, S. F. \& Hylemon, P. B. (1997). Biotransformation of bile acids, cholesterol, and steroid hormones. In Gastrointestinal Microbiology, vol. I, Gastrointestinal Ecosystems and Fermentations, pp. 470-510. Edited by R. I. Mackie \& B. A. White. New York: International Thomson Publishing.

Batta, A. K., Salen, G., Arora, R., Shefer, S., Batta, M. \& Person, A. (1990). Side chain conjugation prevents bacterial 7dehydroxylation of bile acids. J Biol Chem 265, 10925-10928.

Cheah, P. Y. (1990). Hypothesis for the etiology of colorectal cancer - an overview. Nutr Cancer 14, 5-13.

Christiaens, H., Leer, R. J., Pouwels, P. H. \& Verstraete, W. (1992). Cloning and expression of a conjugated bile salt hydrolase gene from Lactobacillus plantarum by using a direct plate assay. Appl Environ Microbiol 58, 3792-3798.

Cole, C. B. \& Fuller, R. (1974). Bile acid deconjugation and attachment of chicken gut bacteria: their possible role in growth depression. Br Poult Sci 25, 227-231.

Coleman, J. P. \& Hudson, L. L. (1995). Cloning and characterization of a conjugated bile acid hydrolase gene from Clostridium perfringens. Appl Environ Microbiol 61, 2514-2520.

Dashkevicz, M. P. \& Feighner, S. D. (1989). Development of a differential medium for bile salt hydrolase-active Lactobacillus spp. Appl Environ Microbiol 55, 11-16.
De Smet, I., Van Hoorde, L., De Saeyer, N., Vande Woestyne, M. \& Verstraete, W. (1994). In vitro study of bile salt hydrolase (BSH) activity of $\mathrm{BSH}$ isogenic Lactobacillus plantarum 80 strains and estimation of cholesterol lowering through enhanced BSH activity. Microb Ecol Health Dis 7, 315-329.

De Smet, I., Van Hoorde, L., Vande Woestyne, M., Christiaens, H. \& Verstraete, W. (1995). Significance of bile salt hydrolytic activities of lactobacilli. J Appl Bacteriol 79, 292-301.

Edgell, D. R., Belfort, M. \& Shub, D. A. (2000). Barriers to intron promiscuity in bacteria. J Bacteriol 182, 5281-5289.

Edwards, J. B. D. M., Ravassard, P., Icard-Liepkalns, C. \& Mallet, J. (1995). cDNA cloning by RT-PCR. In PCR 2: a Practical Approach, pp. 89-118. Edited by M. J. McPherson, B. D. Hames \& G. R. Taylor. New York: Oxford University Press.

Elkins, C. A. \& Savage, D. C. (1998). Identification of genes encoding conjugated bile salt hydrolase and transport in Lactobacillus johnsonii 100-100. J Bacteriol 180, 4344-4349.

Eyssen, H. \& deSomer, P. (1963). The mode of action of antibiotics in stimulating growth of chicks. J Exp Med 117, 127-138.

Gilliland, S. E. \& Speck, M. L. (1977). Deconjugation of bile acids by intestinal lactobacilli. Appl Environ Microbiol 33, 15-18.

Gopal-Srivastava, R. \& Hylemon, P. B. (1988). Purification and characterization of a bile salt hydrolase from Clostridium perfringens. J Lipid Res 29, 1079-1085.

Grill, J.-P., Schneider, F., Crociani, J. \& Ballongue, J. (1995). Purification and characterization of conjugated bile salt hydrolase from Bifidobacterium longum BB536. Appl Environ Microbiol 61, 2577-2582.

Hylemon, P. B. \& Harder, J. (1999). Biotransformation of monoterpenes, bile acids, and other isoprenoids in anaerobic ecosystems. FEMS Microbiol Rev 22, 475-488.

Kandell, R. L. \& Bernstein, C. (1991). Bile salt/acid induction of DNA damage in bacterial and mammalian cells: implications for colon cancer. Nutr Cancer 16, 227-238.

Kawamoto, K., Horibe, I. \& Uchida, K. (1989). Purification and characterization of a new hydrolase for conjugated bile acids, chenodeoxycholyltaurine hydrolase, from Bacteroides vulgatus. J Biochem 106, 1049-1053.

Kay, R. M. (1981). Effects of diet on the fecal excretion and bacterial modification of acidic and neutral steroids, and implications for colon carcinogenesis. Cancer Res 41, 3774-3777.

Kishinaka, M., Umeda, A. \& Kuroki, S. (1994). High concentrations of conjugated bile acids inhibit bacterial growth of Clostridium perfringens and induce its extracellular cholylglycine hydrolase. Steroids 59, 485-489.

Lundeen, S. G. \& Savage, D. C. (1990). Characterization and purification of bile salt hydrolase from Lactobacillus sp. strain 100-100. J Bacteriol 172, 4171-4177.

Lundeen, S. G. \& Savage, D. C. (1992a). Multiple forms of bile salt hydrolase from Lactobacillus sp. strain 100-100. J Bacteriol 174, $7217-7220$.

Lundeen, S. G. \& Savage, D. C. (1992b). Characterization of an extracellular factor that stimulates bile salt hydrolase activity in Lactobacillus sp. strain 100-100. FEMS Microbiol Lett 94, 121-126.

Min, G. \& Powell, J. R. (1998). Long-distance genome walking using the long and accurate polymerase chain reaction. Biotechniques 24, 398-400.

Moser, S. A. \& Savage, D. C. (2001). Bile salt hydrolase activity and resistance to toxicity of conjugated bile salts are unrelated properties in lactobacilli. Appl Environ Microbiol 67, 3476-3480. 
Saier, M. H., Jr, Beatty, J. T., Goffeau, A. \& 11 other authors (1999). The major facilitator superfamily. J Mol Microbiol Biotechnol 2, 257-279.

Savage, D. C. (1977). Microbial ecology of the gastrointestinal tract. Annu Rev Microbiol 31, 107-133.

Savage, D. C. (2000). Probiotic bacteria in the gastrointestinal environment: factors influencing their survival and colonization. Biosci Microflora 19, 9-14.

Savage, D. C., Lundeen, S. G. \& O'Connor, L. T. (1995). Mechanisms by which indigenous microorganisms colonise epithelial surfaces as a reservoir of the lumenal microflora in the gastrointestinal tract. Microecol Ther 21, 27-36.

Stellwag, E. J. \& Hylemon, P. B. (1976). Purification and characterization of bile salt hydrolase from Bacteroides fragilis subsp. fragilis. Biochim Biophys Acta 452, 165-176.
Tanaka, H., Hashiba, H., Kok, J. \& Mierau, I. (2000). Bile salt hydrolase of Bifidobacterium longum - biochemical and genetic characterization. Appl Environ Microbiol 66, 2502-2512.

Thanassi, D. G., Cheng, L. W. \& Nikaido, H. (1997). Active efflux of bile salts by Escherichia coli. J Bacteriol 179, 2512-2518.

Weber, B. A., Klein, J. R. \& Henrich, B. (1998). The arbZ gene from Lactobacillus delbrueckii subsp. lactis confers to Escherichia coli the ability to utilize the $\beta$-glucoside arbutin. Gene 212, 203-211.

Wong, M. H., Oelkers, P., Craddock, A. L. \& Dawson, P. A. (1994). Expression cloning and characterization of the hamster ileal sodium-dependent bile acid transporter. J Biol Chem 269, $1340-1347$.

Received 1 May 2001; revised 15 August 2001; accepted 20 August 2001. 